\title{
Modificación del comportamiento tribológico de zirconio y titanio mediante implantación de nitrógeno
}

\author{
J. A. GARCía(1), A. GUetTe (2), A. MEDRANO (1), C. LABRUGERE ${ }^{(2)}$, M. $\operatorname{RICO}^{(1)}$, \\ M. LAHAYE ${ }^{(2)}$, R. SANCHEZ (1), R.J. RODRÍGUEZ (1) \\ (1) Centro de Ingeniería Avanzada de Superficies AIN, Cordovilla-Pamplona \\ (2) Centre de Caractérisation des Materiaux Avancés31191 87, Av. Du Docteur A. Schweitzer 33680 PESSAC Cedex
}

\begin{abstract}
En el presente trabajo se estudian los efectos introducidos por la implantación de nitrógeno atómico y molecular en Ti y Zr comercialmente puros. Se relacionan los cambios en la dureza superficial y coeficiente de fricción con los cambios en la composición química superficial. En todos los casos se ha encontrado que la implantación iónica proporciona aumentos de dureza que en algunos de los casos llega hasta el $80 \%$. Se encontraron así mismo mejoras en la resistencia al desgaste y disminuciones del coeficiente de fricción de hasta un 50\%. La caracterización AES y XPS pone de manifiesto la aparición de precipitados de nitruros, con mayor concentración para la implantación de altas dosis de nitrógeno atómico sobre titanio.
\end{abstract}

\section{Palabras clave: Implantación iónica, nitrógeno, titanio, circonio, desgaste}

Tribological behaviour of nitrogen implanted into zirconium and titanium.

This paper gathers the studies of the effects introduced by Nitrogen implantation on Ti and Zr. Atomic and molecular Nitrogen have been implanted on Titanium and Zirconium samples to investigate the role of these elements in the nitrides precipitation. In all cases an increase in microhardness, which in some cases was greater than $80 \%$ was found out. Moreover was observed improvements in the wear resistance and a decrease in the friction coefficient. AES and XPS spectroscopy revealed that nitride precipitation in titanium is more significant for the high atomic doses of Nitrogen implantation.

Keywords: Ion implantation, nitrogen, titanium, zirconium, tribology

\section{INTRODUCCIÓN.}

En los últimos años se han dedicado numerosos esfuerzos al estudio de las modificaciones del titanio y sus aleaciones mediante la implantación de iones ligeros (1-3), hecho que refleja la creciente demanda industrial de estas aleaciones. Son bien conocidos los efectos positivos que la implantación iónica de nitrógeno tiene sobre la dureza superficial y la resistencia al desgaste de las aleaciones de titanio(1-3), un hecho ampliamente aprovechado en la industria aeronáutica y biomédica. Por otro lado, también se han realizado numerosos estudios sobre la modificación de las propiedades superficiales mediante la implantación de nitrógeno sobre circonio (4-6). En este artículo se resumen y comentan los efectos y modificaciones producidos por la implantación de distintas dosis de nitrógeno atómico y molecular sobre probetas de titanio y circonio, tratando de relacionar los cambios introducidos en las propiedades tribológicas, con los cambios en la composición superficial de ambos materiales.

\section{PROCEDIMIENTO EXPERIMENTAL}

\subsection{Materiales y preparación.}

Se prepararon probetas de Titanio de $30 \mathrm{~mm}$ de diámetro para los ensayos tribológicos, y de $8 \mathrm{~mm}$ de diámetro para los análisisespectroscópicos, a partir de barras de Ti GR.2 Por la misma razón las probetas de circonio fueron preparadas en tamaños de $20 \mathrm{~mm}$ x $20 \mathrm{~mm}$ y $10 \mathrm{~mm} \times 10 \mathrm{~mm}$, a partir de plancha de $\mathrm{Zr}$ de 99.9 de pureza.

Posteriormente las probetas recibieron un pulido a espejo con
TABLA I. RESUMEN DE LAS IMPLANTACIONES REALIZADAS.

\begin{tabular}{|c|c|c|c|c|}
\hline Substrato & $\begin{array}{c}\text { Especie } \\
\text { Implantada }\end{array}$ & Dosis & Energia & $\begin{array}{c}\text { Profundidad } \\
\text { del pico }\end{array}$ \\
\hline Titanio & $\mathrm{N}^{+}$ & $4 \times 10^{17} \mathrm{at} / \mathrm{cm}^{2}$ & $150 \mathrm{Kev}$ & $0,28 \mu \mathrm{m}$ \\
\hline Titanio & $\mathrm{N}^{+}$ & $8 \times 10^{17} \mathrm{at} / \mathrm{cm}^{2}$ & $150 \mathrm{Kev}$ & $0,28 \mu \mathrm{m}$ \\
\hline Titanio & $\mathrm{N}_{2}^{+}$ & $4 \times 10^{17} \mathrm{at} / \mathrm{cm}^{2}$ & $150 \mathrm{Kev}$ & $0,12 \mu \mathrm{m}$ \\
\hline Titanio & $\mathrm{N}_{2}{ }^{+}$ & $8 \times 10^{17} \mathrm{at} / \mathrm{cm}^{2}$ & $150 \mathrm{Kev}$ & $0,12 \mu \mathrm{m}$ \\
\hline Circonio & $\mathrm{N}^{+}$ & $4 \times 10^{17} \mathrm{at} / \mathrm{cm}^{2}$ & $150 \mathrm{Kev}$ & $0,20 \mu \mathrm{m}$ \\
\hline Circonio & $\mathrm{N}^{+}$ & $8 \times 10^{17} \mathrm{at} / \mathrm{cm}^{2}$ & $150 \mathrm{Kev}$ & $0,20 \mu \mathrm{m}$ \\
\hline Circonio & $\mathrm{N}_{2}^{+}$ & $4 \times 10^{17} \mathrm{at} / \mathrm{cm}^{2}$ & $150 \mathrm{Kev}$ & $0,10 \mu \mathrm{m}$ \\
\hline Circonio & $\mathrm{N}_{2}{ }^{+}$ & $8 \times 10^{17} \mathrm{at} / \mathrm{cm}^{2}$ & $150 \mathrm{Kev}$ & $0,10 \mu \mathrm{m}$ \\
\hline
\end{tabular}

Alúmina de 0.05 micras, y se sumergieron en acetona en un baño de ultrasonidos durante 15 minutos.

\subsection{Tratamientos y Caracterización.}

Las implantaciones se realizaron con un implantador de alta corriente del Centro de Ingeniería Avanzada de Superficies de AIN. Los parámetros más relevantes de las implantaciones se muestran en la Tabla I.

La dureza Universal H.U. de los materiales tratados y sin tratar se ha medido con un Microdurómetro FICHERSCOPE $\mathrm{H} 100$, empleando cargas de $2 \mathrm{mN}, 5 \mathrm{mN}$ y $25 \mathrm{mN}$.

Los ensayos de fricción se han realizado utilizando un Tribómetro FALEX ISC-320PC, con una configuración Ball-ondisk que permite controlar todos los parámetros relevantes, incluida la humedad relativa. Para los ensayos se emplearon bolas de 1/2" de acero 100Cr6. 


\section{RESULTADOS}

\subsection{Espectroscopía AUGER.}

Se realizaron espectros AUGER sobre las probetas tratadas en cada uno de los experimentos, así como sobre probetas de referencia de ambos materiales. Los resultados revelan perfiles de concentración del nitrógeno que se asemejan en gran medida a las simulaciones realizadas con los programas TRIM y PROFILE. En la Figura 1 puede observarse el perfil AUGER para la implantación de $\mathrm{N}^{+}$sobre titanio con dosis de $8 \times 10^{17}$ at. $/ \mathrm{cm}^{2}$.

\subsection{Espectroscopía XPS.}

De cara a estudiar la formación de nitruros, se llevaron a cabo espectros XPS de cada una de las probetas implantadas, así como para las probetas de referencia. Los espectros XPS revelan la existencia de precipitados de $\mathrm{TiN}$ en las probetas de titanio implantadas con nitrógeno. En las Figura 2 y 3 puede observarse el pico $1 \mathrm{~s}$ del nitrógeno a $396.9 \mathrm{eV}$, valor típico del enlace Ti-N $(8,9)$. Estos precipitados se observaron en concentraciones mayores en el caso de la implantación de altas dosis de nitrógeno atómico. En las probetas de circonio implantadas también se observaron precipitados de $\mathrm{ZrN}$.

\subsection{Ensayos de Microdureza.}

La Tabla II recoge los resultados de la dureza para las probetas de titanio y circonio a diferentes niveles de carga final. Puede observarse como a medida que aumentamos la carga final, las modificaciones introducidas por las implantaciones dejan de observarse. Este hecho es debido a que para $5 \mathrm{mN}$ y $25 \mathrm{mN}$ la penetración del indentador supera el espesor de la zona afectada por la implantación.

En los gráficos de las Figuras 4 y 5 pueden observarse las curvas de carga-descarga a $2 \mathrm{mN}$ de carga final. En estas curvas pueden apreciarse los incrementos de dureza para las probetas implantadas. Debe ser tenido en cuenta que las microdurezas medidas a $2 \mathrm{mN}$ de carga final, no son exclusivamente las de la zona modificada por la implantación, ya que para medir la dureza de una capa la profundidad de penetración del indentador no debe superar el 10\% del espesor de la capa. En estos casos las durezas medidas son una mezcla de la dureza de la zona implantada y de la dureza del substrato, por lo que cabría esperar aumentos mayores de la dureza en ensayos con niveles más bajos de carga final. En las figuras 4 y 5 también puede apreciarse que las probetas implantadas con mayor dosis presentan un incremento superior de la microdureza.

\subsection{Ensayos de Fricción.}

En la Tabla III se recogen los valores del coeficiente de fricción en distintas condiciones de carga para las probetas de titanio y circonio. Como puede observarse la implantación iónica de nitrógeno atómico y molecular producen importantes disminuciones del coeficiente de fricción, que en algunos casos supera el $70 \%$ con respecto al material sin implantar.

En las probetas de Zirconio, la reducción del coeficiente de fricción es ligeramente superior para las implantaciones de Nitrógeno atómico, como también puede observarse en la Tabla III y en las Figuras 6 y 7.

Los ensayos de fricción realizados a las probetas de titanio

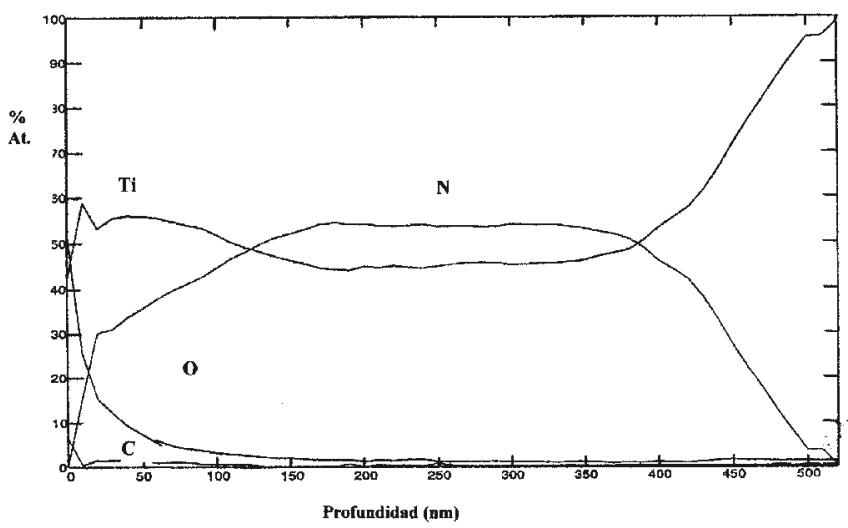

Figura 1. Espectro AUGER para las probetas de Titanio implantadas con $8 \times 10^{17}$ At. $/ \mathrm{cm}^{2}$ de $\mathrm{N}^{+}$.

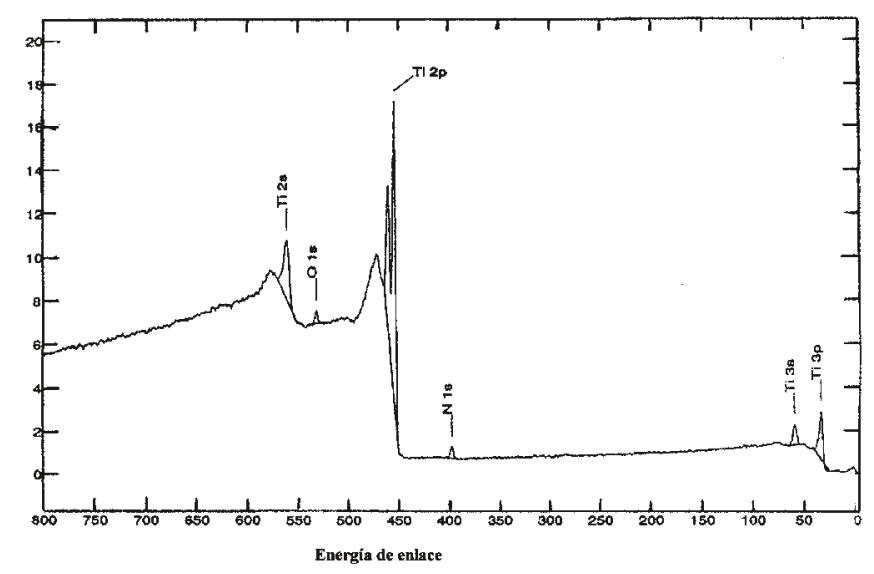

Figura 2. Espectro XPS para las probetas de Titanio implantadas con $8 \times 10^{17}$ At. $/ \mathrm{cm}^{2}$ de $\mathrm{N}^{+}$.

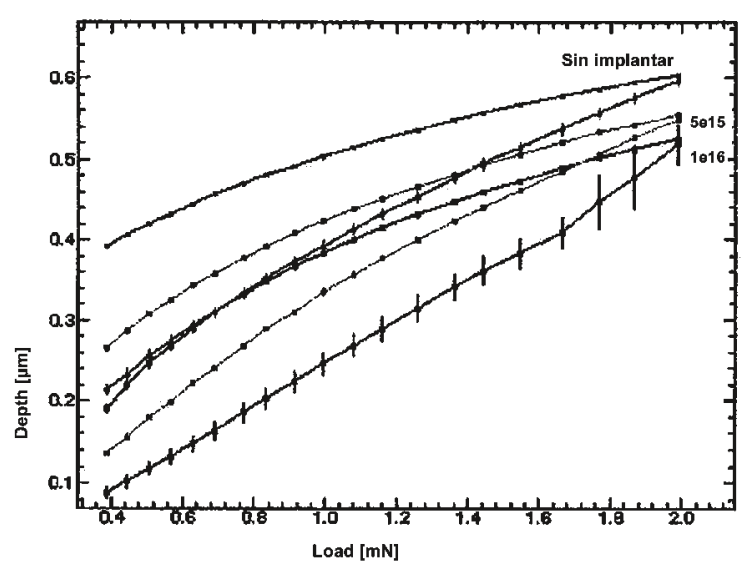

Figura 3. Espectro XPS para las probetas de Titanio implantadas con $8 \times 10^{17}$ At. $/ \mathrm{cm}^{2}$ de ${ }^{\mathrm{N}+}$. 


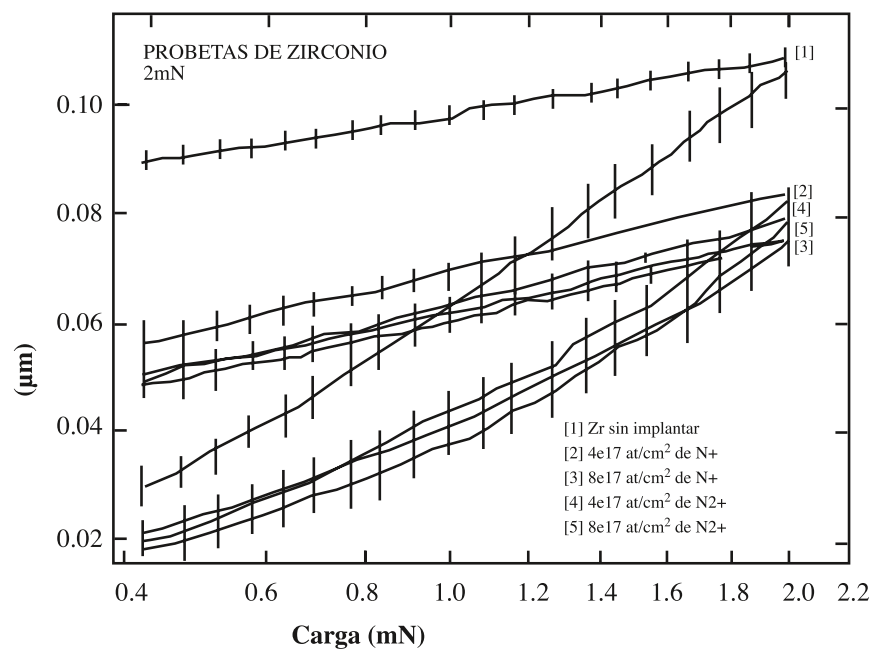

Figura 4. Curvas de carga-descarga a $2 \mathrm{mN}$ de carga final para las probetas de Titanio.

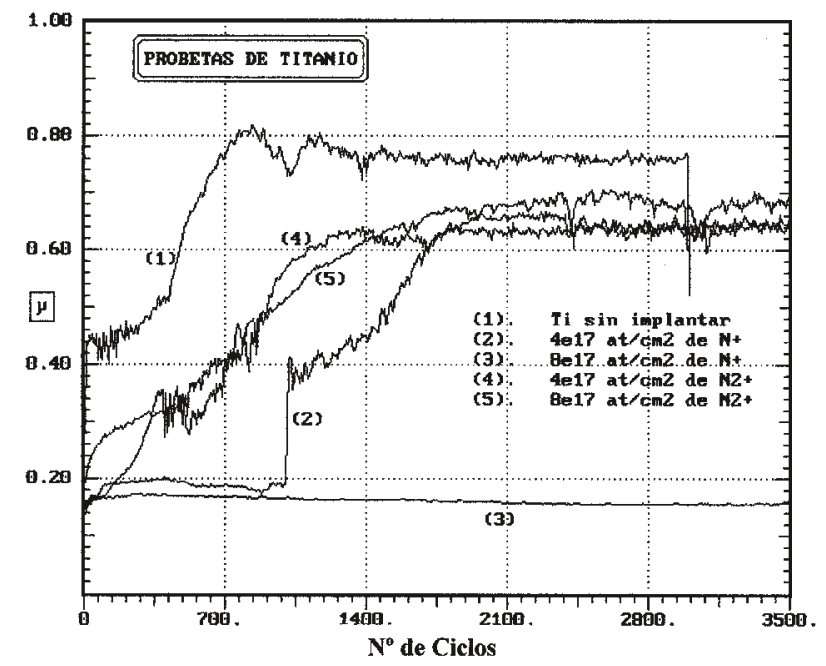

Figura 6. Evolución del coeficiente de fricción para las probetas de Titanio.

TABLA II: DUREZA UNIVERSAL A DISTINTOS NIVELES DE CARGA FINAL.

\begin{tabular}{|c|c|c|c|}
\hline Titanio & \multicolumn{3}{|c|}{ Dureza Universal en $\mathrm{N} / \mathrm{mm}^{2}$ a cargas finales de: } \\
\hline Dosis & $2 m N$ & $5 \mathrm{mN}$ & $25 \mathrm{mN}$ \\
\hline Sin implantar & $2.71 I$ & 2.832 & 2.442 \\
\hline $4 \times 10^{17} \mathrm{a} / \mathrm{cm}^{2}$ de $N^{+}$ & 4.426 & 2.989 & 2.322 \\
\hline $8 \times 10^{l 7} \mathrm{a}^{\prime} \mathrm{cm}^{2}$ de $N^{+}$ & 4.457 & 3.714 & 2.522 \\
\hline $4 \times 10^{17} \mathrm{at} / \mathrm{cm}^{2} \mathrm{de} \mathrm{N}_{2}^{+}$ & 3.896 & 3.555 & 2.630 \\
\hline $8 \times 10^{17} \mathrm{at}^{\mathrm{c}} \mathrm{cm}^{2} \mathrm{de} \mathrm{N}_{3}^{+}$ & 4.175 & 3.111 & 2.423 \\
\hline Circonio & \multicolumn{3}{|c|}{ Dureza Untversal en $\mathrm{N} / \mathrm{mm}^{2}$ a cargas finales de } \\
\hline Dosis & $2 m N$ & $5 \mathrm{mN}$ & $25 \mathrm{mN}$ \\
\hline Sin implantar & 2.450 & 2.495 & 2.195 \\
\hline $4 \times 10^{17} \mathrm{at}^{\mathrm{cmm}}$ de $N^{+}$ & 4.230 & 2.858 & 2.255 \\
\hline $8 \times 10^{17} \mathrm{at}^{\prime} \mathrm{cm}^{2} \mathrm{de} \mathrm{N}^{+}$ & 5.005 & 3.395 & 2.219 \\
\hline $4 x 10^{17} a t / c^{2} d e N_{2}^{+}$ & 4.290 & 2.630 & 2.121 \\
\hline $8 \times 10^{17} \mathrm{at} / \mathrm{cm}^{2}$ de $\mathrm{N}_{2}{ }^{+}$ & 4.607 & 3.141 & 2.063 \\
\hline
\end{tabular}

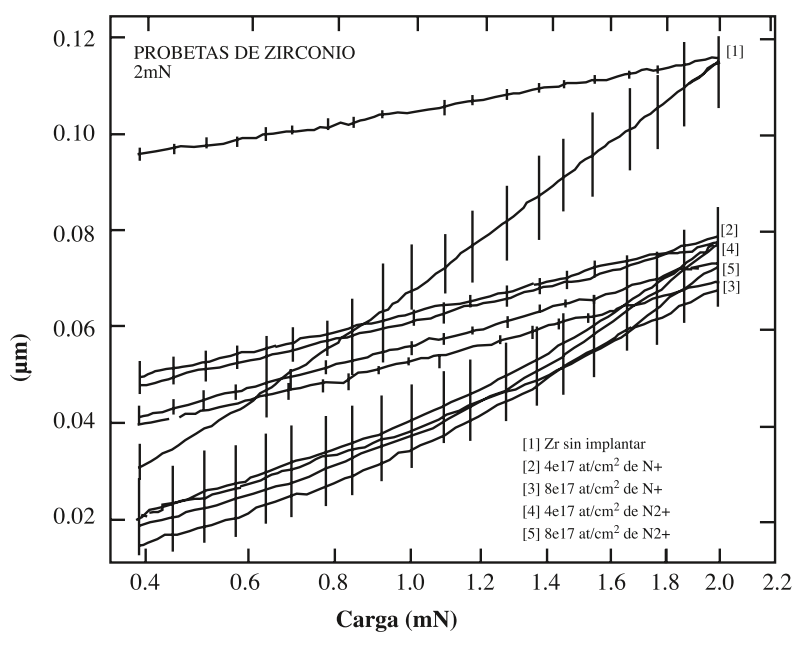

Figura 5. Curvas de carga-descarga a $2 \mathrm{mN}$ de carga final para las probetas de Zirconio.

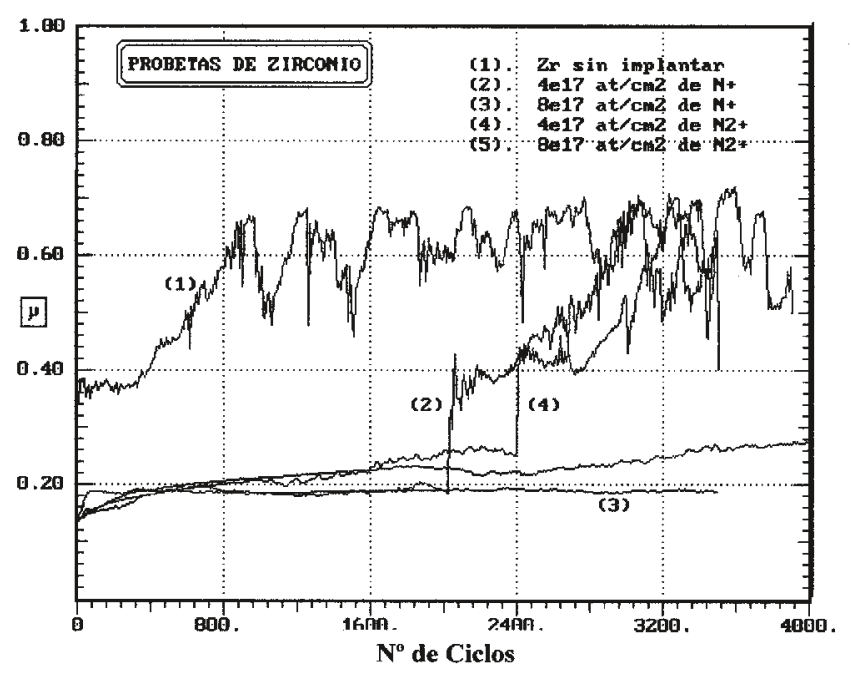

Figura 7. Evolución del coeficiente de fricción para las probetas de Zirconio.

TABLA III: COEFICIENTE DE FRICCIÓN A DISTINTOS NIVELES DE CARGA.

\begin{tabular}{|c|c|c|c|c|c|}
\hline \multicolumn{6}{|c|}{ Probetas de Titanio } \\
\hline Carga & $\operatorname{Ref} T i$ & $\begin{array}{c}4 \times 10^{17} \\
a v / \mathrm{cm}^{2} \text { de } N^{+}\end{array}$ & $\begin{array}{c}8 \times 10^{17} \\
a \nu / \mathrm{cm}^{2} \text { de } N^{+} \\
\end{array}$ & $\begin{array}{c}4 \times 10^{17} \mathrm{av} \mathrm{cm}^{2} \\
\mathrm{de} \mathrm{N}_{2}{ }^{+} \\
\end{array}$ & $\begin{array}{c}8 \times 10^{17} \mathrm{at} / \mathrm{cm}^{2} \\
\operatorname{de~} \mathrm{N}_{2}^{+}\end{array}$ \\
\hline $100 \mathrm{~g}$ & $\ldots$ & $0,2 \rightarrow 0,7$ & 0,2 & 0,7 & 0,7 \\
\hline $50 \mathrm{~g}$ & $-\cdots$ & $0,2 \rightarrow 0,7$ & 0,2 & $0,2 \rightarrow 0,7$ & 0,7 \\
\hline $50 \mathrm{~g}$ & 0,8 & 0,2 & 0,2 & 0,6 & 0,7 \\
\hline $50 \mathrm{~g}$ & 0,8 & $0,2 \rightarrow 0,7$ & 0,16 & 0,7 & 0,6 \\
\hline \multicolumn{6}{|c|}{ Probetas de Zirconio } \\
\hline Carga & $\operatorname{Ref} Z r$ & $\begin{array}{c}4 \times 10^{17} \\
a t / \mathrm{cm}^{2} \text { de } N^{+} \\
\end{array}$ & $\begin{array}{c}8 \times 10^{17} \\
a t / \mathrm{cm}^{2} \text { de } N^{+} \\
\end{array}$ & $\begin{array}{c}4 x 10^{17} \mathrm{av}^{\prime} \mathrm{cm}^{2} \\
\mathrm{deN}_{2}^{+}\end{array}$ & $\begin{array}{c}8 \times 10^{17} \mathrm{at} / \mathrm{cm}^{2} \\
\mathrm{de} \mathrm{N}_{2}^{+}\end{array}$ \\
\hline $100 \mathrm{~g}$ & 0,7 & $0,2 \rightarrow 0,7$ & 0,2 & $0,25 \rightarrow 0,7$ & 0,30 \\
\hline $50 \mathrm{~g}$ & 0,7 & $0,2 \rightarrow 0,7$ & 0,2 & 0,25 & 0,25 \\
\hline $50 \mathrm{~g}$ & 0,7 & $0,2 \rightarrow 0,7$ & 0,2 & 0,20 & 0,25 \\
\hline $50 \mathrm{~g}$ & 0,7 & 0,16 & 0,16 & 0,25 & 0,20 \\
\hline
\end{tabular}


implantadas con nitrógeno atómico muestran un coeficiente de fricción centrado en torno a 0.2 que se mantiene en estos valores durante más de 20.000 ciclos. Estos bajos valores son debidos a la precipitación de $\mathrm{TiN}$ y $\mathrm{TiO}_{2}$ como ha quedado reflejado en los espectros de XPS. Por otro lado para las implantaciones con nitrógeno molecular la capa formada se desprende aproximadamente una vez superados los 1000 ciclos de ensayo.

El aumento del coeficiente de fricción desde 0.2 hasta 0.7 , es debido al desgaste de la zona afectada por la implantación. Como puede observarse en la Tabla III, esta situación es más frecuente para las implantaciones con dosis más bajas de nitrógeno atómico.

\section{CONCLUSIONES}

La implantación de altas dosis de nitrógeno atómico y molecular ( $>4 \times 10^{17}$ at. $\left./ \mathrm{cm}^{2}\right)$, produce aumentos de hasta un $70 \%$ en la dureza superficial del titanio. Estos aumentos de dureza son mayores para las dosis más altas, tanto para el nitrógeno atómico como para el molecular.

La implantación de nitrógeno atómico y molecular a altas dosis sobre circonio, revela aún mayores aumentos de dureza superficial que para el titanio, que en los mejores casos supera el 75\% con respecto al material sin implantar. También para el circonio son mayores los aumentos de dureza para las dosis más altas $\left(8 \times 10^{17}\right.$ at. $\left./ \mathrm{cm}^{2}\right)$.

La implantación de altas dosis $\left(8 \times 10^{17}\right.$ at. $\left./ \mathrm{cm}^{2}\right)$ de $\mathrm{N}+$ sobre titanio disminuye el coeficiente de fricción más de un $60 \%$, y se mantiene estable durante más de 20.000 ciclos.
El coeficiente de fricción de las probetas de circonio implantadas con nitrógeno disminuyó en más de un $60 \%$. En este caso no se encontraron diferencias entre las implantaciones a dosis medias y altas.

Los espectros XPS y AUGER revelan la nucleación de precipitados de TiN responsables del incremento de dureza superficial en las probetas de Ti, así como la nucleación de $\mathrm{ZrN}$ en las probetas de $\mathrm{Zr}$ implantadas con nitrógeno.

\section{BIBLIOGRAFÍA}

1. F. Torregrosa, L. Barrallier, L. Roux.. Phase analysis, microhardness and tribological behaviour of Ti-6Al-4V after ion implantation of nitrogen in connection with its application for hip-joint prosthesis. Thin Solid Films 266 245-253 (1995).

2. J. Rieu, A. Pichat, L.M. Rabbe, C. Chabrel and M. Robelet. Deterioration mecanisms of joint prosthesis materials. Biomaterials, 11 51-54 (1990).

3. Jukka Lausmaa, Tord Röstlund and Harry Mckellop. A surface spectroscopic study of nitrogen ion-implanted $\mathrm{Ti}$ and $\mathrm{Ti}-6 \mathrm{Al}-4 \mathrm{~V}$ wear against UHMWPE. Surf. Interface Anal. Vol 15, 328-336 (1989).

4. Soji Miyagawa et al.Composition and structure of zirconium implanted with nitrogen at high fluence. Nucl. Instr. And Meth, B80/81 480-484 (1993).

5. Y. Etoh, S. Shimada and H. Takahashi.. Surface improvement of $\mathrm{Zr}$ and Zircaloy-2 by metal implantation J. Nucl. Mater. 202 122-136 (1993).

6. I. Takano, S. Isobe, T. A. Sasaki and Y. Baba High nitrogen ion-implantation into zirconium. Appl. Surf. Sci. 3725 (1989).

7. H. Hochst, R.D. Bringans, P. Teiner and Th. Wolf, UPS studies of nitrogen ion implanted zirconium Phys. Rev. B, 257183 (1982).

8. S. Badrinarayaman, S. Sinha and A.B. Mandale. XPS studies of nitrogen ion implanted zirconium and titanium J. Electr. Spectr. And Phen., 49 303-309 (1989). 